\title{
EVALUACIÓN DE LA COMPRENSIÓN ORAL EN INGLÉS AL FINAL DEL PRIMER CICLO DE LA EDUCACIÓN SECUNDARIA OBLIGATORIA CONFORME A LOS OBJETIVOS DEL DISEÑO CURRICULAR BASE ANDALUZ ${ }^{1}$
}

\author{
$\mathrm{M}^{\mathrm{a}}$ Dolores Corpas Arellano ${ }^{2}$
}

\begin{abstract}
Resumen: Esta investigación tiene como objetivo conocer el nivel de comprensión oral en inglés que alcanza el alumnado de segundo de Educación Secundaria Obligatoria (ESO) al término del primer ciclo de la misma. Para llevar esto a cabo, hemos partido de los objetivos expuestos en el Diseño Curricular Base andaluz (decreto 148/2002 de mayo, BOJA ${ }^{\circ} 75$ ). Según los resultados del alumnado, ofrecemos su ubicación conforme al Marco Común Europeo.

Concluimos diciendo que nuestro alumnado consigue poco más de la mitad de los objetivos evaluados. A tenor de los resultados, afirmamos que su nivel se sitúa en el nivel A2 inicial según el Marco Común Europeo.

Palabras clave: Evaluación, comprensión oral, ESO, objetivos.

Abstract: This research aims to know the English comprehension level that the students have in the Second year ${ }^{3}$ of Compulsory Secondary Education (CSE) when they finish its first stage. In order to carry this out, we have taken the objectives in the Andalusian Core Curricular Design (decree 148/2002, May, BOJA n. 75). According to our students' results, we want to classify them taking into account the Common European Framework. We conclude stating that our students get little more than half of the evaluated objectives. Bearing these results in mind, we admit that our students' English level is A2 initial in the Common European Framework classification.
\end{abstract}

Key words: Evaluation, listening comprehension, CSE, objectives.

\section{INTRODUCCIÓN}

A diferencia de la escritura y la lectura, la audición y el habla son naturales al ser humano. De hecho, aprendemos nuestra lengua materna oyendo y sin esfuerzo aparente. El input oral permite que el individuo reproduzca sonidos, que unidos forman palabras. De igual manera, parece obvio afirmar que el input oral es esencial para aprender una lengua.

La comunicación oral es un proceso complejo y cambiante. Para que una comunicación oral sea efectiva, se necesita que estén presentes varios aspectos tanto en el hablante como

Fecha de recepción: febrero 2008.

Fecha de aceptación y versión final: junio 2008.

2 Profesora de Enseñanza Secundaria, I.E.S. Americo Castro, Huetor Tajar (Granada); $\square$ mariolacorpas@ wanadoo.es.

314 years old. 
en el oyente. Así el hablante debe querer hablar, además de tener un propósito comunicativo y desear que algo suceda como resultado de lo que dice. Por último, elige la lengua que considera que es más apropiada con respecto a su intención.

En lo que se refiere al oyente de una lengua, éste debe querer oír algo, debe estar interesado en lo que se está diciendo y, lo que es aún más importante debe estar preparado para procesar, exactamente, la información que se le está enviando, con el objeto de evitar malentendidos.

A toda esta complejidad de la compresión oral añadimos que el estudiante de una lengua extranjera debe enfrentarse a otros problemas, según Ur (1984) el identificar sonidos distintos de sus lenguas maternas, la comprensión de una entonación y acentuación diferente, concentrarse en el mensaje oral neutralizando sonidos e interferencias, predecir el mensaje e intencionalidad del hablante, comprender el vocabulario y estructuras y vencer el cansancio del oyente. Estos obstáculos dificultan enormemente la comprensión oral en una lengua extranjera.

Como profesionales de la enseñanza de la lengua inglesa en Educación Secundaria Obligatoria, nos preguntamos qué nivel de inglés desarrolla nuestro alumnado al terminar el primer ciclo de este nivel educativo con respecto a los objetivos del Diseño Curricular Base Andaluz, actualmente vigentes, o sea, los recogidos en el decreto 148/2002 de mayo $\left(\right.$ BOJA $\left.^{\circ} 75\right)$. En cuanto a la enseñanza de lenguas extranjeras, y al desarrollo de la comprensión oral, el apartado j del mismo decreto declara que el alumnado debe "comprender y producir mensajes orales y escritos contextualizados, en la lengua o lenguas extranjeras objeto de estudio" (BOJA de 27 de junio de 2002: 11470). ${ }^{4}$

\section{LA COMPRENSIÓN ORAL}

\subsection{Características de la compresión oral}

La audición y el habla dependen mutuamente. Así, en la conversación, según van Lier (1996: 167), el encuentro comunicativo más típico, las dos destrezas comparten protagonismo. Cuando un individuo habla, otro debe escuchar para comprender.

Tradicionalmente, tanto la lectura como la escritura se han considerado destrezas receptivas, de comprensión e incluso pasivas. Conforme a Harris y Hewitt (2005: 284), esta creencia ha sido reemplazada por una perspectiva que valora la comprensión escrita y oral como habilidades literarias y oratorias, aunque utilizan medios para comunicarse distintos. Nos referimos al papel y al sonido. La comprensión escrita limitada por el espacio, la escrita por el tiempo.

Como características de la comprensión oral, McDonough (1998) señala las siguientes:

- El medio es el sonido. De ahí que tenga una duración pasajera a diferencia del medio escrito. Además el oyente no tiene control sobre la velocidad del input.

\footnotetext{
4 Recientemente, el título I, en el capítulo III, artículo 23 expone los objetivos de la LOE (Ley Orgánica 2/2006 de 3 de mayo, publicada en el BOE n 106, el 4 de mayo), para la Educación Secundaria Obligatoria. En lo que al apartado de lenguas extranjeras se refiere, el punto i) expone:"ii) Comprender y expresarse en una o más lenguas extranjeras de manera apropiada (BOE, 4 de mayo de 2006:17169)”.
} 
- La información presentada en los mensajes orales tiende a ser menos densa y más redundante y repetitiva que en la expresión oral. La gramática y el discurso son menos elaborados. Por ejemplo, las oraciones no suelen ser complejas, los mecanismos de cohesión más simples, etc.

- El discurso oral se caracteriza por las dudas, pausas, oraciones a medio completar, cambios de tema y de estilo.

- El habla normalmente va acompañado de elementos suprasegmentales, rasgos paralingüísticos y no paralingüísticos como la entonación, el tono de voz y los gestos, que ayudan a la comprensión y son parte de la formulación de los actos de habla. No todo son ventajas. También el oyente debe hacer frente a sonidos ajenos al mensaje que le ocupa. Estos sonidos, diversos en forma y origen, interfieren una adecuada recepción de la producción oral.

- Los mensajes tradicionales son cooperativos. Se construyen conjuntamente por los hablantes y los oyentes mientras cambian de rol en una conversación, gracias a la interacción.

A todo esto debemos añadir que la comprensión oral es un fenómeno psicológico (Field 2003). Desde el punto de vista cognitivo, además de un fenómeno social (Carrier 1999), donde interviene el entorno y los participantes. Nunan (2002) agrega que también es un fenómeno fisiológico puesto que interviene el sistema auditivo del ser humano.

\subsection{Recursos y técnicas para el desarrollo de la comprensión oral en lengua inglesa}

Tradicionalmente, para la enseñanza de la comprensión oral se ha utilizado la técnica de dividir las actividades según el momento de su realización, es decir, anterior a la audición (pre-listening), durante la audición (listening) y después de la misma (post- listening).

Según Field (2002), esta división, aunque todavía en vigor, ha transformado las características de sus actividades. Así en la etapa anterior a la audición, se solía enseñar todo el vocabulario nuevo del texto. En cambio, la tendencia actual es a contextualizar la audición tal y como ocurre en un contexto cotidiano de la vida real (McDonough: 1995), ofreciendo en términos de van Dijk (1981), la posición de los hablantes (estatus, roles, etc.), la propiedades (el sexo, edad, etc.), las relaciones entre los mismos (la autoridad, el dominio, etc.) $y$, finalmente, las funciones (jefe, juez, hermano, vecino, etc.).

La etapa de la audición se caracteriza por ser o bien extensiva o intensiva. Conforme con Field (2002), la primera está relacionada con un periodo inicial de audición extensiva que permite al oyente acostumbrarse a las características de la voz de hablante, como su tono, velocidad y calidad de voz. La audición intensiva, obviamente, menos extensa que la anterior, se suele grabar y repetir varias veces. Se intenta reproducir la audición tal y como se produce la vida real.

Como Harris y Hewitt (2005) apuntan, las actividades de después de la audición conviene clasificarlas en dos grupos. Unas examinan un aspecto particular del texto escuchado detenidamente. La otra se utiliza como trampolín para realizar otra actividad, como utilizar el tema para, según Hedge (2000) actividades de comprensión escrita, expresión escrita u oral. 
Brown (2001) expone una serie de técnicas para la enseñanza de la comprensión oral clasificadas por niveles para la comprensión de textos orales. A continuación hacemos referencia al nivel inicial de los tres propuestos (inicial, intermedio y avanzado) por este autor, por ser el que más se ajusta a nuestro alumnado:

a) Discriminación de entonación dentro de los límites de la oración.

b) Discriminación entre fonemas.

c) Audición selectiva para detectar finales morfológicos.

d) Reconocimiento de palabras.

e) Audición para detectar el orden de las palabras en una oración normal.

f) Discriminar entre reacciones emocionales.

g) Emparejar una oración con una imagen.

h) Proporcionar la idea general.

i) Reconocer el tema.

j) Construir una red semántica de asociaciones de palabras.

k) Reconocer una palabras familiar y relacionarla con una categoría.

1) Seguir una dirección.

\subsection{La comprensión oral según el marco común europeo}

El Marco Común Europeo de las Lenguas (2001: 65-66) especifica el tipo de actividades que un usuario de la lengua como oyente recibe y procesa. Estas actividades incluyen la comprensión oral de anuncios públicos como información, advertencias e instrucciones, comprensión oral de textos emitidos por los medios de comunicación, radio, televisión, grabaciones y cine, comprensión oral como miembros de una audiencia en teatros, reuniones públicas, audiciones públicas o entretenimiento, además de la comprensión oral en conversaciones relativas a la vida cotidiana. Así, el propósito del individuo puede ser: extraer una idea general, extraer información específica, una comprensión minuciosa, etc.

En cuanto a la evaluación de la comprensión oral, el Marco Común Europeo ofrece escalas para la comprensión oral global, comprensión oral de la interacción entre hablantes nativos, comprensión oral como miembro de una audiencia en directo y comprensión oral de emisiones de medios de comunicación y de grabaciones. Además, divide en seis los niveles de comprensión oral, desde el nivel más bajo A1 hasta el más alto C2. Reproducimos a continuación los dos niveles más bajos con respecto a la comprensión oral (Marco Común Europeo 2001: 66). 
Fig. 1: Niveles iniciales del Marco Común Europeo en comprensión oral.

\begin{tabular}{|l|l|}
\hline \multirow{2}{*}{ A2 } & $\begin{array}{l}\text { Puede comprender lo suficiente para solucionar los problemas de una cuestión concreta } \\
\text { siempre que la producción oral sea clara y articulada lentamente. }\end{array}$ \\
\cline { 2 - 3 } & $\begin{array}{l}\text { Puede comprender frases y expresiones relacionadas con áreas de prioridad inmediata } \\
\text { (por ejemplo, información personal y familiar muy básica, compras, geografía local, } \\
\text { empleo) siempre que la producción oral sea clara y articulada lentamente. }\end{array}$ \\
\hline A1 & $\begin{array}{l}\text { Puede seguir la producción oral que sea muy lenta y articulada muy cuidadosamente, con } \\
\text { pausas largas para que pueda asimilar el significado. }\end{array}$ \\
\hline
\end{tabular}

\section{ALGUNOS ESTUDIOS SOBRE LA EVALUACIÓN DE LA COMPRENSIÓN ORAL EN LENGUA INGLESA EN EDUCACIÓN SECUNDARIA OBLIGATORIA}

El INCE 5 publicó un informe titulado La Evaluación de la Enseñanza y el Aprendizaje de la Lengua Inglesa en Educación Secundaria Obligatoria 2001. En síntesis, los objetivos generales son conocer y valorar las capacidades básicas logradas por el alumnado de la Educación Secundaria Obligatoria (ESO), al termino del primer y segundo ciclo, con respecto al nivel de comunicación, conocimientos de la lengua inglesa adquiridos y conocer los factores del proceso enseñanza-aprendizaje que influyan en los resultados del alumnado.

Centrándonos en segundo de ESO, la prueba incluye la evaluación de las destrezas receptivas (lectura y audición), de las destrezas productivas (el habla y la escritura), reflexión sobre la lengua y aspectos socioculturales. Los porcentajes de cada prueba se distribuyen de la siguiente manera: la comprensión oral y escrita constituye el 50\%, la expresión escrita el $15 \%$ y la oral el $20 \%$. Por último, las pruebas concernientes a la reflexión sobre la lengua representan el 10\% y los aspectos socioculturales el 5\%. Estos dos tipos de conocimientos se han evaluado por preguntas contextualizadas o por textos utilizando la escritura y la lectura.

131 preguntas constituyen el total de la prueba, si se exceptúan las de expresión oral, y están distribuidas en cuatro modelos. Dichos modelos comparten 21 preguntas. Las demás son específicas de cada uno.

Según los datos de la comprensión oral, la media alcanza el 56\%, siendo las de comprensión por reorganización la que ha conseguido un mayor porcentaje $(68 \%)$, mientras que la asociación llega al 54\%. Un 56\% obtiene la identificación de la información específica.

\section{DISEÑO DEL ESTUDIO}

\subsection{Objetivos}

Este estudio pretende dar respuesta a los siguientes objetivos científicos:

A. Conocer qué nivel de comprensión oral en lengua inglesa desarrolla el alumnado al término del primer ciclo de la Enseñanza Secundaria Obligatoria con respecto a los objetivos del Diseño Curricular Base Andaluz. Debido a las características de los

Ahora denominado INECSE. 
propios objetivos, hemos podido evaluar la comprensión oral en los objetivos dos, cinco, seis, ocho, nueve y diez recogidos en el decreto 148/2002 de mayo (BOJA $\mathrm{n}^{\mathrm{o}} 75$ ), que seguidamente reproducimos:

Objetivo 2. Comprender de forma global y específica textos orales y escritos en lengua extranjera, emitidos por hablantes en situaciones habituales de comunicación o por los medios de comunicación.

Objetivo 5. Transferir al conocimiento de la lengua extranjera las estrategias de comunicación adquiridas en la lengua materna o en el aprendizaje de otras lenguas, con el fin de realizar tareas y como instrumento para el desarrollo de la autonomía.

Objetivo 6. Reflexionar sobre el funcionamiento lingüístico y comunicativo de la lengua extranjera como instrumento facilitador y regulador del aprendizaje de la misma y para mejorar las producciones personales y contribuir a la adquisición autónoma del idioma.

Objetivo 8. Reflexionar sobre los propios procesos de aprendizaje y desarrollar interés por incorporar mejoras que lleven al éxito en la consecución de las tareas planteadas.

Objetivo 9. Apreciar y reaccionar ante los usos imaginativos y creativos de la lengua extranjera a partir de narraciones, poemas, canciones, películas, etc., para estimular la propia creatividad.

Objetivo 10. Apreciar los significados sociales y culturales que transmiten las lenguas extranjeras, en tanto que suponen otras formas de codificar la realidad, de establecer las relaciones sociales e interpersonales, ayudan a eliminar prejuicios y estereotipos y a afirmar y relativizar la identidad cultural propia.

B. Ubicar al alumnado conforme al Marco Común Europeo.

\subsection{Población}

La investigación se ciñe a un Instituto de Educación Secundaria andaluz. La prueba ha sido realizada por cincuenta y tres alumnos/as de segundo de Educación Secundaria Obligatoria.

\subsection{Instrumentos para la recogida de datos}

Seguidamente, describimos las tareas ${ }^{6}$ (véase anexos I y II) que ha realizado el alumnado de nuestra investigación, no sin antes apuntar que las pruebas fueron realizadas al final del tercer trimestre, a pocas semanas de terminar el primer ciclo de la Educación Secundaria Obligatoria.

\footnotetext{
6 Estas tareas han sido extraídas de libros de texto autorizados por el Ministerio de Educación y actualmente en vigor.
} 


\section{Tarea 1}

Objetivo que evalúa: Comprender de forma general y específica textos orales emitidos en lengua inglesa.

Formato: El alumno debe responder a una serie de cuestiones tras haber oído un texto oral. Extensión de los textos: Unas cien palabras.

Número de preguntas: Cuatro preguntas.

Duración: Entre cinco y siete minutos.

\section{Tarea 2}

Objetivo que evalúa: Transferir al conocimiento de la lengua inglesa las estrategias de comunicación adquiridas en la lengua materna o en otras lenguas.

Formato: El alumno debe ordenar las oraciones de un texto oral, apoyándose en su conocimiento previo sobre los textos dialógicos.

Extensión de los textos: Unas cuarenta palabras.

Número de preguntas: Seis.

Duración: Unos cinco minutos.

\section{Tarea 3}

Objetivo que evalúa: Reflexionar sobre el funcionamiento lingüístico y comunicativo de la lengua inglesa.

Formato: Tras haber oído una serie de oraciones, el alumno debe identificar las grafías con los fonemas de las palabras seleccionadas.

Extensión de los textos: Siete palabras.

Número de preguntas: Siete.

Duración: Tres minutos.

\section{Tarea 4}

Objetivo que evalúa: Reflexionar sobre los propios procesos de aprendizaje y desarrollar interés por introducir mejoras.

Formato: El alumno debe completar los huecos de un texto, tras haberlo oído y teniendo en cuenta las palabras dadas.

Extensión de los textos: Cincuenta palabras.

Número de preguntas: Cinco.

Duración: Unos tres o cuatro minutos.

\section{Tarea 5}

Objetivo que evalúa: Apreciar y reaccionar ante los usos imaginativos y creativos de la lengua inglesa.

Formato: El alumno debe completar una serie de oraciones que faltan en un texto, tras haberlo oído.

Extensión de los textos: Cincuenta y cuatro palabras.

Número de preguntas: Tres.

Duración: Unos cinco minutos. 


\section{Tarea 6}

Objetivo que evalúa: Apreciar los significados sociales y culturales que transmite la lengua inglesa.

Formato: El alumno debe completar los huecos de un texto, tras haberlo oído. El texto representa una conversación telefónica. El alumno debe conocer los mecanismos de comunicación en esa situación.

Extensión de los textos: Treinta y cinco palabras.

Número de preguntas: Cuatro.

Duración: Unos tres o cuatro minutos.

Fig.2: Distribución de ítems con respecto a los objetivos que evalúan.

\begin{tabular}{|c|c|c|c|c|c|c|}
\hline Objetivo & Obj 2 & Obj 5 & Obj 6 & Obj 8 & Obj 9 & Obj 10 \\
\hline Items & $1,2,3,4$ & $\begin{array}{l}5,6,7,8, \\
9,10\end{array}$ & $\begin{array}{l}11,12,13, \\
14,15,16, \\
17,\end{array}$ & $\begin{array}{l}18,19,20, \\
21,22\end{array}$ & $23,24,25$ & $\begin{array}{l}26,27,28, \\
29\end{array}$ \\
\hline
\end{tabular}

\subsection{Criterios de corrección}

Muy buen oyente/ Buen oyente: Comprende todo lo que ha sido tratado en el currículo. Entiende la mayor parte de lo estudiado. Puede omitir algo que en ningún caso afecta seriamente a la comprensión del mensaje.

Oyente Medio: Sólo comprende los puntos fundamentales. Pierde detalles y datos específicos que no impiden seguir la conversación, el texto o el mensaje.

Mal Oyente/ Muy Mal Oyente: Sólo comprende algunas palabras aisladas y frases. Es incapaz de comprender el mensaje o texto a no ser que se le repita varias veces y muy lentamente. Sólo comprende unas pocas palabras aisladas. Es incapaz de comprender oraciones $\mathrm{y} / \mathrm{o}$ mensajes aunque se le repita.

\subsection{Tratamiento estadístico}

El programa informático que se ha utilizado es SPSS 11.01, por medio de cual ha sido posible hallar la desviación típica, media y porcentaje que se expone en cada uno de los objetivos.

\subsection{Validez y fiabilidad}

Puesto que las pruebas han sido diseñadas, elaboradas, pasadas y evaluadas por varios expertos, la validez de las pruebas están amparadas por un juicio de expertos.

En cuanto al modelo Alpha de Cronbach, un 0’805, nos indica una buena fiabilidad. 


\section{RESULTADOS DE COMPRENSIÓN ORAL EN SEGUNDO DE ESO}

A. Nivel de comprensión oral en lengua inglesa del alumnado al término de segundo de ESO

La media de aciertos de la compresión oral ha sido 3'11 objetivos correctos de los seis objetivos que se han evaluado (objetivos 2, 5, 6, 8, 9 y 10). Un 25\% del alumnado ha superado menos de dos objetivos, un 50\% menos de 3 objetivos y un 75\% menos de 4 objetivos.

Objetivo 2. Comprender de forma global y especifica textos orales y escritos en lengua extranjera, emitidos por hablantes en situaciones habituales de comunicación o por los medios de comunicación.

De los cincuenta y tres alumnos que han realizado la prueba, treinta de ellos han logrado superar este objetivo. Esto representa el 56'6\% de la muestra.

Objetivo 5. Transferir al conocimiento de la lengua extranjera las estrategias de comunicación adquiridas en la lengua materna o en el aprendizaje de otras lenguas, con el fin de realizar tareas y como instrumento para el desarrollo de la autonomía.

Este objetivo es el que ha logrado un mayor porcentaje de aciertos. El 67'9\% del alumnado supera este objetivo. Así, de los cincuenta y tres alumnos, treinta y seis logran superarlos.

Objetivo 6. Reflexionar sobre el funcionamiento lingüistico y comunicativo de la lengua extranjera como instrumento facilitador y regulador del aprendizaje de la misma y para mejorar las producciones personales y contribuir a la adquisición autónoma del idioma.

A diferencia del objetivo anterior, éste es el que peores resultados consigue. Tan sólo dieciséis alumnos superan este objetivo, lo que representa el 30’2\% del total.

Objetivo 8. Reflexionar sobre los propios procesos de aprendizaje y desarrollar interés por incorporar mejoras que lleven al éxito en la consecución de las tareas planteadas.

Un 56'6\% del alumnado logra este objetivo. Son 30 los alumnos que logran resultados positivos en este objetivo.

Objetivo 9. Apreciar y reaccionar ante los usos imaginativos y creativos de la lengua extranjera a partir de narraciones, poemas, canciones, películas, etc., para estimular la propia creatividad.

De los cincuenta y tres alumnos, sólo veinte tienen éxito en la consecución del objetivo 9. Esto representa al $37^{\prime} 7 \%$ del alumnado.

Objetivo 10. Apreciar los significados sociales y culturales que transmiten las lenguas extranjeras, en tanto que suponen otras formas de codificar la realidad, de establecer las relaciones sociales e interpersonales, ayudan a eliminar prejuicios y estereotipos y a afirmar y relativizar la identidad cultural propia.

Este objetivo lo consiguen treinta y tres de los cincuenta y tres alumnos que conforman la muestra. Por lo que, podemos afirmar que un 62 ’3\% logran superarlo. 
La distribución de aciertos según los objetivos se expresa así:

Comprensión oral

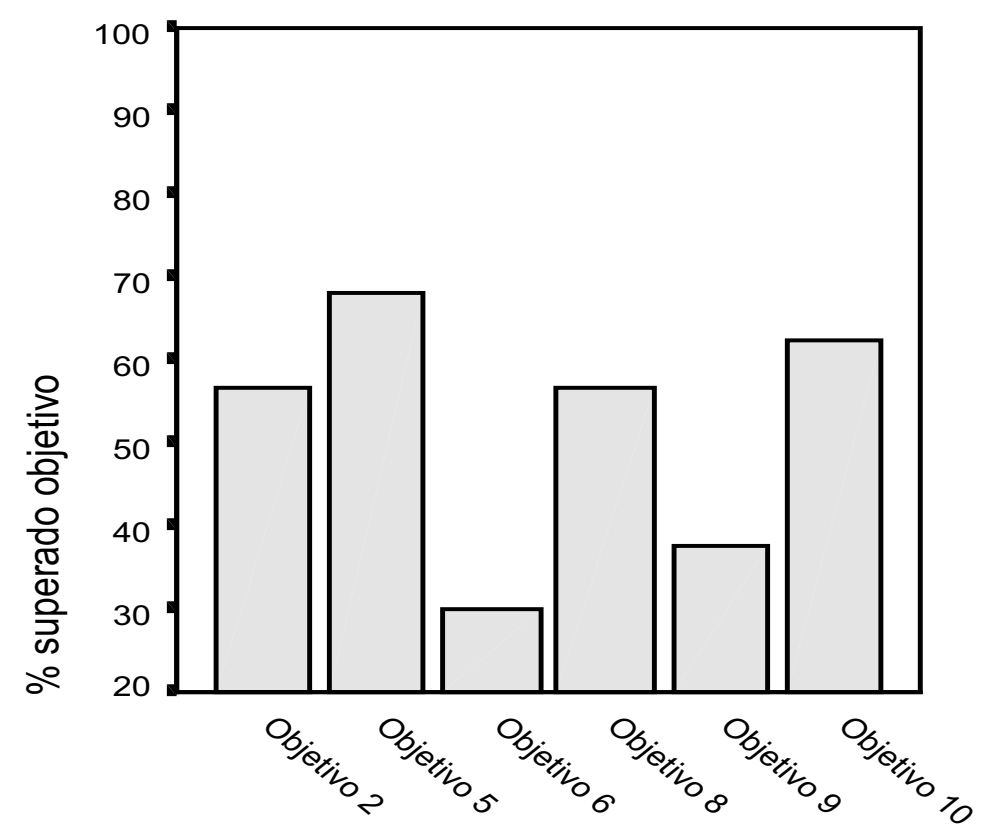

Fig 3: Resultados en Compresión Oral (Segundo ESO) según cada objetivo.

No todos los resultados han conseguido una puntuación similar. Los gráficos de abajo representan por un lado, un objetivo superado por un amplio número y por otro lado, un objetivo superado por un bajo número de estudiantes.

a) Gráfico con alto porcentaje de aciertos

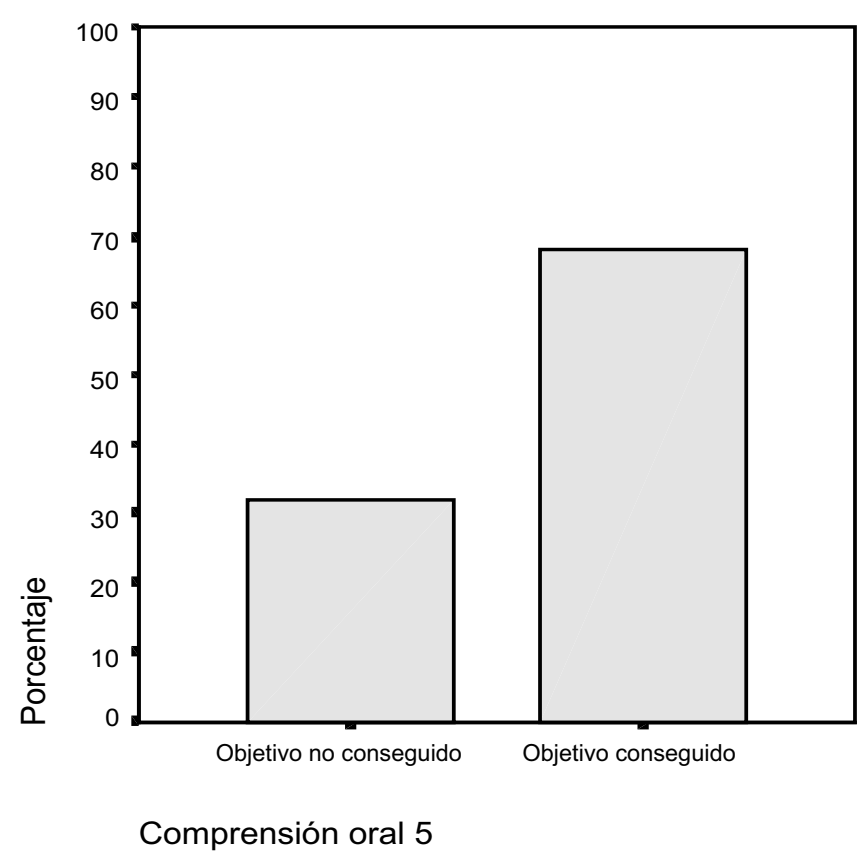


b) Gráfico con bajo porcentaje de aciertos

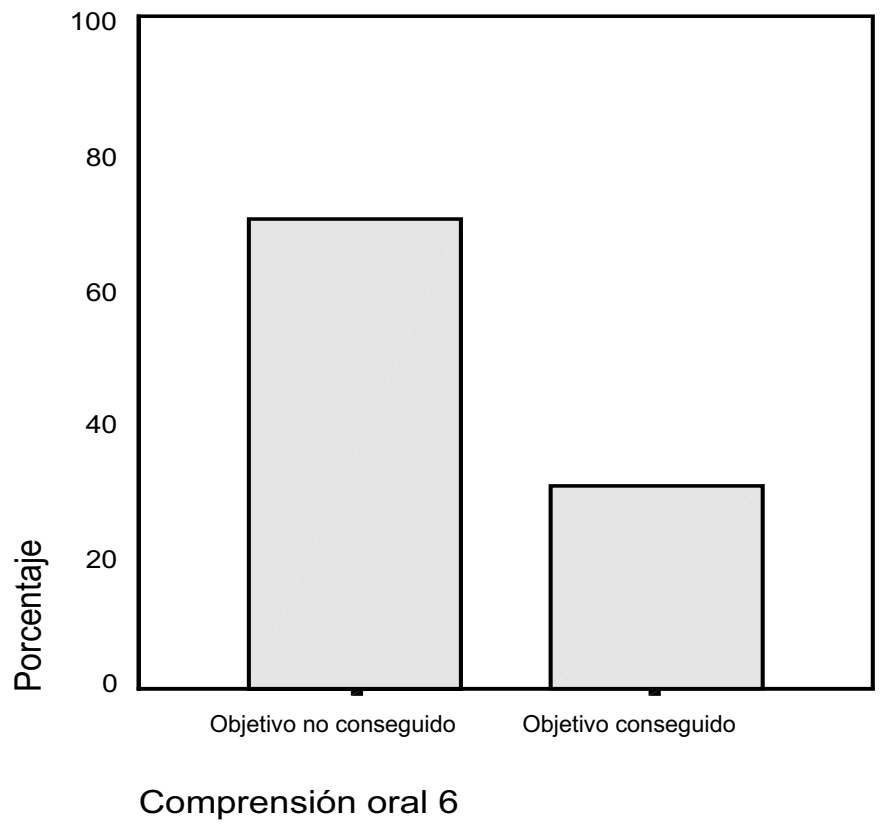

B. Ubicar al alumnado conforme al Marco Común Europeo

A tenor de los resultados, el alumnado medio se ubica en el nivel A2 inicial del Marco Común Europeo, es decir, puede, siempre que la producción sea clara y lenta, comprender frases y expresiones relacionadas con temas de prioridad inmediata.

\section{CONCLUSIONES}

El alumnado ha superado sólo 3'11 objetivos de los seis evaluados, en otras palabras, prácticamente la mitad de los objetivos de la prueba. Aún más preocupante es que un $25 \%$ del alumnado haya superado menos de dos objetivos.

El alumnado medio puede transferir al conocimiento de la lengua extranjera las estrategias de comunicación adquiridas en la lengua materna y apreciar los significados sociales y culturales que transmiten la lengua inglesa. Es capaz de ordenar y completar palabras extraídas de un diálogo siguiendo un texto oral.

Tiene alguna dificultad para comprender de forma global y específica textos orales en lengua inglesa, emitidos por hablantes en situaciones habituales de comunicación o por los medios de comunicación, además de tener algún problema para reflexionar sobre los propios procesos de comunicación. Presenta dificultades para responder a preguntas sobre un texto oral y completar un texto.

No obstante, no es capaz de reflexionar sobre el funcionamiento lingüístico y comunicativo de la lengua inglesa. Tampoco aprecia ni reacciona ante los usos imaginativos y creativos en lengua inglesa. Es incapaz de identificar grafías sin pronunciación en inglés.

Con respecto a la clasificación del Marco Común Europeo de las Lenguas, podemos situar al alumnado medio en el nivel A 2 inicial, es decir, puede comprender frases y expre- 
siones relacionadas con áreas de prioridad inmediata (por ejemplo, información personal y familiar muy básica, compras, geografía local, empleo) siempre que la producción oral sea clara y articulada lentamente. A esto tenemos que añadir que los textos sean simples y cortos.

\section{REFERENCIAS BIBLIOGRÁFICAS}

CARRIER, K. 1999: "The social environment of second language listening: Does status play a role in comprehension?" Modern Language Journal, 83: 65-79.

Consejo De Europa. 2001: Marco Común Europeo para las Lenguas. http://www.mec. es/programas_europeos/jsp/plantilla.jsp?id=343

Decreto 148/2002 de 14 de mayo, enseñanzas correspondientes a la Educación Secundaria Obligatoria en Andalucía BOJA 27 de junio.

FIELD, J. 2003: "Promoting perception: Lexical segmentation in L" listening". ELT Journal, 57, 4: 325-334.

Harris, T. y E. HewitT 2005: "Listening comprehension”. TELF in Secondary Education. Eds. N. McLaren, D. Madrid, y A. Bueno. Granada: Universidad de Granada, 281-320.

Hedge, T. 2000: Teaching and Learning in the Language Classroom. Oxford: Oxford University Press.

McDonough, S. 1998: “Teaching listening”, Encyclopedic Dictionary of Applied Linguistics. Eds. K. Johnson, y H. Johnson. Massachusetts: Blackwell, 324-330.

NunAN, D. 2002: "Listening in language learning", Methodology in Language Teaching: An Anthology of Current Practice. Eds. J. C. Richards y W. A. Renandya. Cambridge: Cambridge University Press, 238-241.

Real Decreto 1631/2006 de 29 de diciembre, enseñanzas mínimas correspondientes a la Educación Secundaria Obligatoria BOE 5 de enero de 2007.

UR, P. ed. 1984: Teaching Listening Comprehension. Cambridge: Cambridge University Press.

VAn DiJk, T. A. 1981: Studies in the Pragmatics of Discourse. The Hague: Mouton.

VAN LIER, L. 1996: Interaction in the Language Curriculum: Awarenesss, Autonomy and Authenticity. New York: Longman. 
ANEXO I

Pruebas de $2^{\circ}$ de E.S.O. : Compresión Oral

\section{Listening $2^{\text {nd }}$ ESO}

Name:

Class:

School:

Date:

\section{Listen and answer the questions (Obj. 2):}

(1) What's Clyde's job?

(2) How many days a week does he work?

(3) What time does he finish his work?

(4) What does he wear when he's working?

2. Listen and put the sentences into the right order (Obj. 5):

Example $\underline{B}$

(9)

(5)

(6)

(7)

(8) (10)
A) It's 367951 .
B) Where do you leave, please?
C) What's your telephone number?
D) At 11, Hight Street.
E) I'm... Really! Don't be impertinent!
F) And... Er... How old are you?
G) So sorry, lady! 
3. Some letters in certain words are not pronounced. Listen and circle them (Obj. 6):
11. WHEN
12. WRAP
13. LIGHT
14. TALK
15. WOULD
16. NIGHT
17. WALK

4. Listen and complete the gaps (Obj. 8):

Improve Feel Try Enjoy Effort

Your attitudes and motivation in the English class are very important. If you
don't (18)_ interested, if you don't make a considerable
$(19) \ldots$ if you don't $(20) \ldots$ your English. So (22)_ the English class,
you will not $(21) \ldots$ to change
your attitudes and you will notice the results!

\section{Listen and write down the missing sentences (Obj. 9):}

\section{Summer Rain}

Your smile is more beautiful than a summer sky.

(23)

Than the sea your voice is gentler than a butterfly.

(24)

Than a tree.

I know you're feeling lonely and sad again.

And the sky is grey.

But this is only summer rain.

(25)

\section{Listen to the dialogue and complete the gaps (Obj. 10):}

Peter: Hello, Pinner 67358.

Penny: (26) . This is Penny. Is Barbara in?

Peter: Who?

Penny: Barbara. Is (27) in? 
Peter: Hold on a minute. Barbara is for (28)

Barbara: (29)

Peter: You're welcome!

\section{ANEXO II}

Pruebas de $2^{\circ}$ de E.S.O.: Transcripción de las pruebas de comprensión oral

\section{Scripts (listening 2nd ESO)}

1.

Clyde is a hairdresser. He works in a unisex hair salon in London. It's one of the most expensive salons in Britain. He works six days a week, from Monday to Saturday. Every morning he starts work at nine o'clock, and he finishes at five. He doesn't wear a uniform, but he always wears nice, smart clothes.

He usually has about four clients each day. Sometimes famous pop stars come into the salon. His most famous client is Céline Dion. Clyde likes talking to his clients about their holidays and families.

2.

- Where do you live, please?

- $\quad$ At 11, High Street.

- $\quad$ And what's your telephone number?

- $\quad$ It's 367951

- $\quad$ And... er... how old are you?

- I'm... Really! Don't be impertinent!

- $\quad$ So sorry, lady!

3.

1. When I was young, I lived in New Cork.

2. Wrap this gift as soon as you can.

3. Could you turn the light on, please?

4. Derek doesn't want to talk to you. He's very angry. 
5. Natalie would like to leave her town and live abroad.

6. How many hours do you sleep each night?

7. Susan and her sister walk five miles each day.

4.

Your attitudes and motivation in the English class are very important. If you don't feel interested, if you don't make a considerable effort to lear, if you don't enjoy the English class, you will not improve your English. So try to change your attitudes and you will notice the results!

5.

\section{SUMMER RAIN}

Your smile is more beautiful than a summer sky.

Your eyes are deeper than the sea.

Your voice is gentler than a butterfly.

Your spirit is stronger than a tree.

I know you're feeling lonely and sad again.

And the sky is grey.

But this is only summer rain.

Tomorrow is a brighter day.

6.

Peter: Hello, Pinner 67358.

Penny: Hello! This is Penny. Is Barbara in?

Peter: Who?

Penny: Barbara. Is she in? Hold on a minute: Barbara! It's for you!

Barbara: Thanks.

Peter: You're welcome. 